\title{
Enhanced Independent Components Analysis (EICA)
}

\author{
Mustafa Almahdi Algaet \\ Department of Computer, Faculty of Education, \\ Elmergib University, Libya
}

\author{
Ali Ahmed Milad \\ Department of Computer, \\ Faculty of Education, Elmergib University, Libya
}

\author{
Abd Samad bin Hasan Basari \\ Department of System and Computer \\ Communication, \\ Faculty of Information and Communication \\ Technology \\ Universiti Teknikal Malaysia Melaka Durian \\ Tunggal Malaysia
}

\author{
Salem H. Almadhun \\ Department of Computer, \\ Faculty of Education, Elmergib University, Libya
}

\begin{abstract}
This paper presents the methodology and description of algorithms involved in this research. This study utilized two different modules a compression module and transceiver module for medical data processing. The first module processes compression and decompression of medical data for storage by using four different algorithms DCT, SPIHT, JPEG 2000, and enhanced independent components analysis (EICA). The second module consists of transceiver simulation module over noisy WLAN-IEEE 802.11b. Compressed medical data using the four algorithms were considered input data stream in transmission phase. Received data stream via WLAN IEEE 802.11 b was decompressed and evaluated.
\end{abstract}

\section{Keywords}

EICA, compression and decompression, WLAN IEEE $802.11 \mathrm{~b}$ and medical data

\section{INTRODUCTION}

Figure 1 illustrates primary steps of the proposed research framework used in medical data simulation followed by a detailed explanation of the used algorithms and techniques applied to medical data to achieve the main objective. Data transmission transfers data from one location to another and stores data for future purposes. In cases of medical data, quality of medical data must be considered despite their growing data size. Therefore, the required compression approach must be performed before transmitting data with acceptable performance quality. 


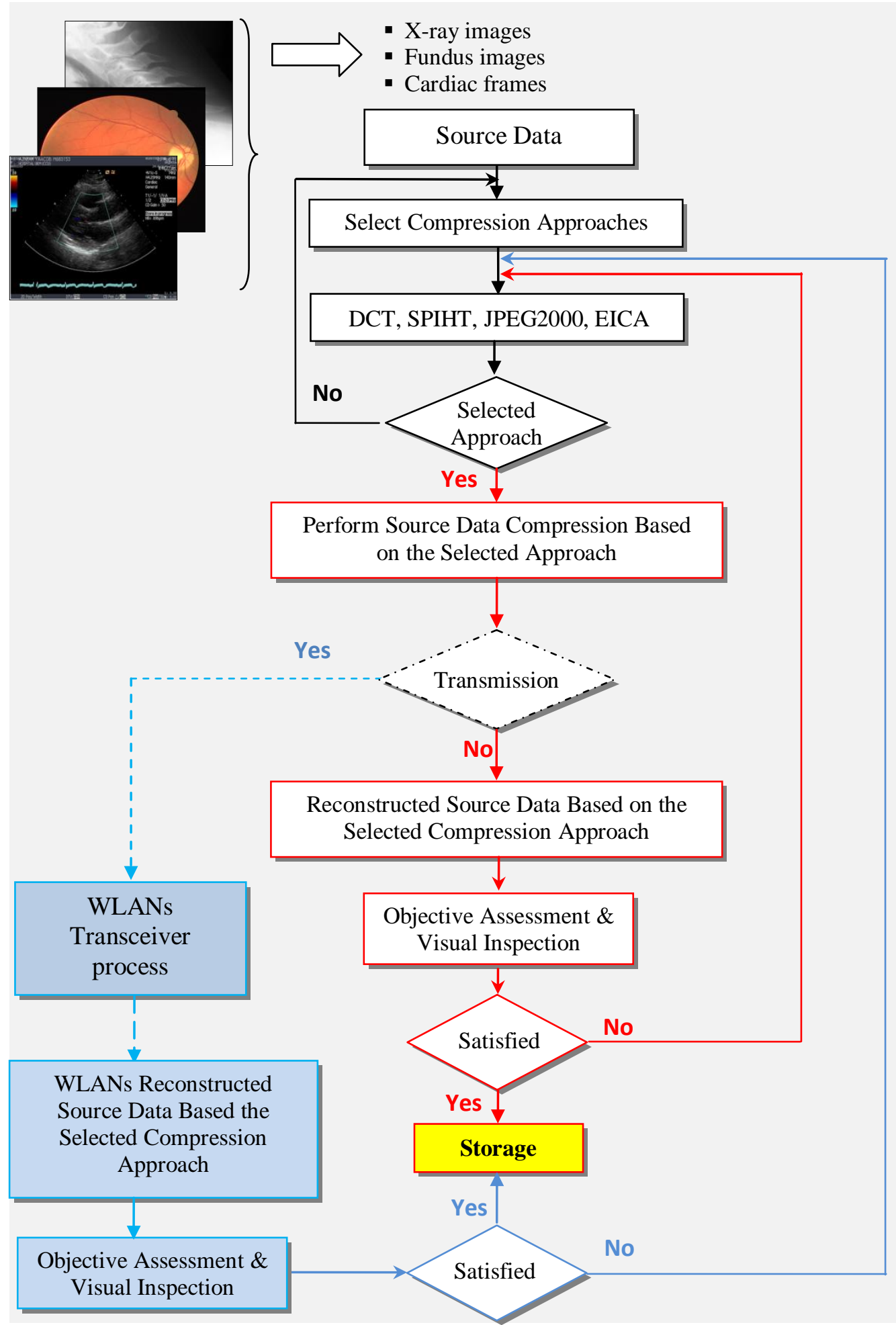

Figure 1: Proposed research frameworks

As shown in Figure 1, the main steps of the proposed framework scheme and detailed explanation of the used algorithms and techniques applied to achieve the main objective are as follows:

1- Compression approach: This approach consists of source data (X-ray images, Fundus images, and Cardiac ultrasound frames) compression with the selected approach includes DCT, SPIHT, JPEG2000 and EICA, followed by a source data reconstruction based the selected compression approach along with an objective and subjective assessment of the resulting data. The resulting data will be stored ones the assessment results satisfied the end user, else the process returned to the compression approach.

2- WLANs Compression approach: This module deals with the WLANs transmission process using the WLANs approach to transfer the source data compressed based the selected compression approach, 
followed by a WLANs reconstructed source data based the selected compression approach accompanied by an objective and subjective assessment of the resulting data. Satisfaction condition based the assessment results are considered as an indicator for the WLANs resulting data storage.

\section{TYPE OF MEDICAL IMAGE}

Medical images are multi-modal and each modality exposes functional anatomical information of various body parts. Each modality has its individual set of conditions such as format resolution, files dimensionality, image production, and acquisition techniques[1]. It may be necessary to create and generate images with diverse modalities such as Computer Tomography (CT), Magnetic Resonance Imaging (MRI), and $\mathrm{X}$-ray images for a proper pathological diagnosis of the same patient [2].

Digital Imaging and Communications in Medicine (DICOM) [3], [4] is the familiar file format for medical images. It consists of additional information related to image modality, acquisition device, and patient identification with raw image data. Two-dimensional DICOM images are larger in terms of size compared to the more common image formats (JPEG, GIF, TIF, etc.).

\subsection{X-ray Images}

The database used in this research consists of 100 images for each digital X-ray images includes cervical, lumbar, and hand, which were collected from the Second National Health and Nutrition Examination Survey (NHANES II), which is maintained by the Lister Hill National Center of Biomedical Communications in the National Library of Medicine (NLM) at the National Institutes of Health (NIH). This collection bears significance in research on the prevalence of osteoarthritis and musculoskeletal diseases. Figure 3.2 illustrates three samples of X-ray images from the database.

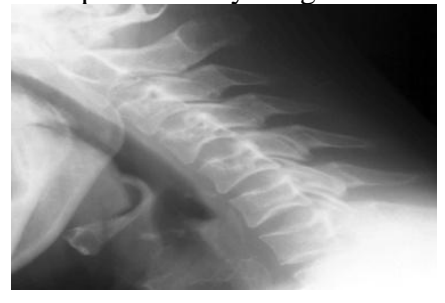

Figure 2: Samples of $X$-ray images

\subsection{Fundus Images}

Only a few public online fundus image databases are available. For example, the common database used for vessel segmentations contains low-resolution images, as shown in Figure 3, which was captured 10 to 15 years ago[5]. The selected database contains 100 fundus images were collected from source web (https://www.opsweb.org/page/fundusimaging),(https://www5 .cs.fau.de/research/data/fundus-images/). Images were anonymous and showed mainly healthy eyes and eyes with diabetic retinopathy.

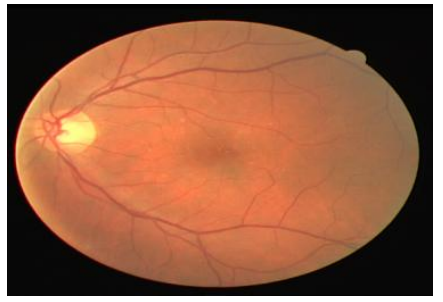

Figure 3: Samples of fundus images

\subsection{Cardiac Ultrasound Images}

Ultrasound measurement depends on numerous properties that differ in propagating sounds; these properties include propagated velocity, decrease, phase shift, and acoustic impedance mismatch. With variation of these properties while propagating through variety of substances, tissue structure characteristics can be analyzed [6]. High-frequency sonic signals above audible frequency range are propagated in fluid and soft tissues. Reflected ultrasonic signals are echoed during image formation. When striking a dense normal tissue, many signals are reflected to create a light image. Thus, images of organs and structures with varying gray shades can be created. The selected database contains 100 Cardiac Ultrasound Images were collected from (www.ultrasoundcases.info/), (www.ob-ultrasound.net/frames.htm). Figure 3 shows samples of ultrasound cardiac video frames.

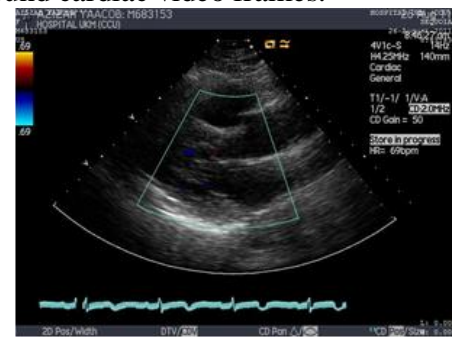

Figure 4: Samples of cardiac ultrasound image under different diagnosis conditions

\section{TRADITIONAL INDEPENDENT COMPONENT ANALYSIS (ICA)}

Independent Component Analysis employs higher-order statistics in projecting data into a new subspace, whose vectors are self-governing statistically. This technique was effectively applied in image processing and psychometric measurements, among other applications [7]. Traditional ICA algorithms use unsupervised learning algorithm that models observed data set as a linear mixture of several mutually exclusive independent, non-Gaussian densities [8]. This modeling approach assumes that there is no advance knowledge of the number of sources involved in the mixing data. This assumption makes it difficult to differentiate between those sources that contribute in the formulation of the mixing image and those that generate noises.

Therefore, conventionally, ICA has been performed in the noise-less limit [9] with noise often being dealt with as an extra source. Independent Component Analysis (ICA) considers a class of probabilistic models in which an observed random vector is obtained according to

$$
S=W X \quad S=\left(s_{1}, s_{1}, s_{1}, \ldots \ldots, s_{n}\right)^{T}
$$

Where $W$ is an $N \times M$ unknown mixing matrix and $X$ 
is a vector of independent sources [9]. The standard goal of ICA is to learn $\mathrm{W}$ from a set of samples of the random vectors. To apply ICA to image, each sample of S usually contains the pixels of an image block. It has been found that images of natural scenes are well modeled when the columns of $\mathrm{W}$, which can be seen as a (possibly over complete) basis as wavelet - like filters, and the independent sources (elements of $X$ ) have heavy tailed distribution [9]. This means that, with high probability, only a small fraction of components of $X$ have significant values, this sparse nature of $X$ underlies the potential usefulness of over complete ICA to compression.

This model is applied to each RGB components values of images of each $X$ sample, which frequently comprises image block pixels. ICA-based compression includes four steps, as follows:

- Step 1: Learn ICA basis vectors from training images with application of FastICA.

- Step 2: Calculate basis matrix inverse (or pseudo inverse), which is referred to as obtained matrix $W$.

- Step 3: Preprocess images to be compressed. First, split images into small square blocks of 8 by 8 . Every block is a matrix $X_{0}$ column. Then, detract the local mean and decrease dimensionality using principal component analysis. Matrix $X$ is eventually obtained.

- $\quad$ Step 4: Obtain independent source $S$ based on the following expression:

$$
S=W . X
$$

With $W$ and $X$ are attained using Steps 2 and 3, respectively.

- Step 5: Compression step is continuous, and zero coefficient percentage is set. Reserves are solely elements with significant $S_{0}(i, j)$ values, whereas other values are set as 0 utilizing the following expression:

$$
\begin{cases}S_{0}(i, j) & \text { if } S_{0}(i, j) \geq 0 \\ 0 & \text { if } S_{0}(i, j)<0\end{cases}
$$

\section{- Quantization}

Output coefficient of ICA is compressed using quantization. Quantization pertains to reducing the number of

\section{- Data Encoding}

Matrices of quantization are encoded for final compression[11]. All elements of $S Q$ are converted by the encoder to a stream of binary data $(0011001010 \ldots)$. This task is achieved using the zigzag sequence shown in Figure 6. The advantage lies in consolidation of relatively large runs of zeros, which compress well. The sequence shown in Figure 6 continues for the entire $8 \times 8$ block .

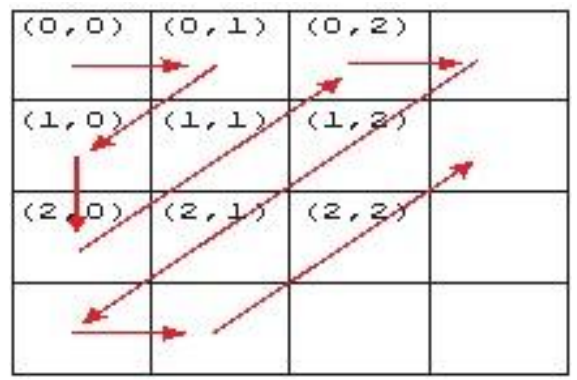

Figure 6: The Zig-Zag Encoder bits required in storing an integer value as it reduces accuracy of integers[10]. Based on ICA coefficient matrix, accuracy of coefficients decreases the farther they move away from DC coefficients. As a result, when moved farther from DC coefficients, elements contribute less to graphical images. Consequently, maintaining precise values of elements becomes less of a concern. In this stage, quantization is achieved by dividing each ICA matrix $(S)$ element by consistent quantization matrix element and then rounding to the nearest integer value, as follows:

$$
?=\operatorname{round}\left(\frac{\left(-1+2^{Q}\right)\left(S-S_{\min }\right)}{S_{\max }-S_{\min }}\right)
$$

where $Q$ refers to number of quantization $(S)$, and $S_{\min }$ and $S_{\max }$ denote minimum and maximum values of ICA matrices, respectively.

Quantization $Q$ values of 8 and 16 are investigated in this research. Using Equation (4), each element in ICA matrix $S Q$ falls in the range of 0 to 255 . Figure 5 shows examples of ICA matrices before and after quantization. The formula shows that smaller ICA coefficients divided by range of ICA coefficient values most often result in low coefficients being rounded down to 0 .

\begin{tabular}{|c|c|c|c|c|c|c|c|}
\hline 92 & 3 & -9 & -7 & 3 & -1 & 0 & 2 \\
\hline-39 & -58 & 12 & 17 & -2 & 2 & 4 & 2 \\
\hline-84 & 62 & 1 & -18 & 3 & 4 & -5 & 5 \\
\hline-52 & -36 & -10 & 14 & -10 & 4 & -2 & 0 \\
\hline-86 & -40 & 49 & -7 & 17 & -6 & -2 & 5 \\
\hline-62 & 65 & -12 & -2 & 3 & -8 & -2 & 0 \\
\hline-17 & 14 & -36 & 17 & -11 & 3 & 3 & -1 \\
\hline-54 & 32 & -9 & -9 & 22 & 0 & 1 & 3 \\
\hline
\end{tabular}

(a)

\begin{tabular}{|c|c|c|c|c|c|c|c|}
\hline 255 & 128 & 110 & 113 & 128 & 122 & 123 & 126 \\
\hline 67 & 40 & 140 & 148 & 120 & 126 & 129 & 126 \\
\hline 3 & 212 & 125 & 97 & 128 & 129 & 116 & 130 \\
\hline 49 & 72 & 109 & 143 & 109 & 129 & 120 & 123 \\
\hline 0 & 66 & 193 & 113 & 148 & 115 & 120 & 130 \\
\hline 34 & 216 & 106 & 120 & 128 & 112 & 120 & 123 \\
\hline 99 & 143 & 72 & 148 & 107 & 128 & 128 & 122 \\
\hline 46 & 169 & 110 & 110 & 155 & 123 & 125 & 128 \\
\hline
\end{tabular}

Figure 5: Example of The ICA Matrices, (a) Before and (b) After Quantization Process

Figures 7 (a) and 7 (b) show examples of ICA matrices before and after encoding previous data. Output of this process comprises $1 \times n$ matrices that contain a stream of data. In this simulation, each element is converted to a binary form using 8-bit data. 


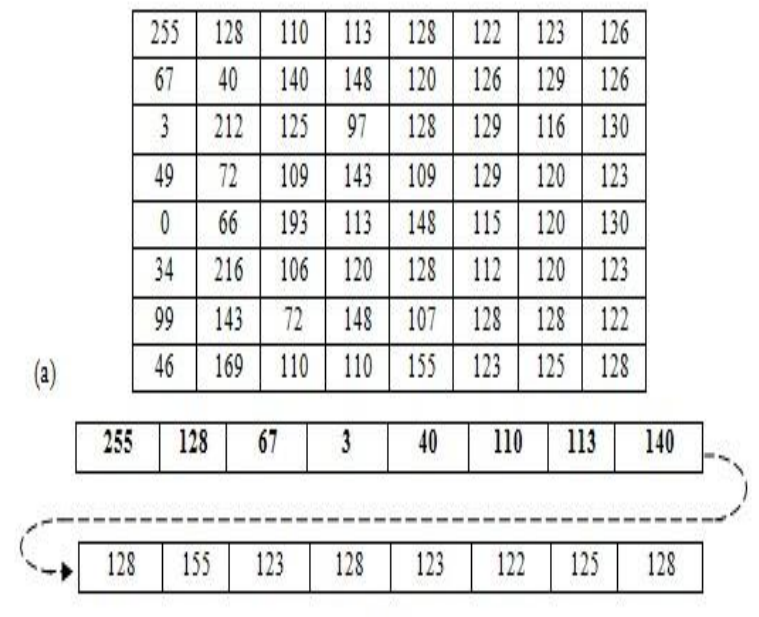

(b)

Figure 7: Example of The ICA Matrices, (a) Before and (b) After Encoding Process

\section{- Image Reconstruction}

After transmission through Wi-Fi protocol connection, new bit stream data are obtained [12]. Inverse quantization and encoding methods are used to encode bit stream data and produce new independent source $S^{*}$. Image $X^{*}$ is reconstructed according to the following equation:

$$
X^{*}=A S^{*}+E\left(X^{o}\right)
$$

Where; $A$ and $E\left(X^{\circ}\right)$ represent parameters computed using basis data of trained image. These parameters are shared between transmitter and receiver of medical data[13]

\section{ENHANCED INDEPENDENT COMPONENT ANALYSIS (EICA)}

A new technique for image compression transforms the image data by block-based ICA. The proposed technique utilises Fast Independent Component Analysis (FastICA) algorithm [14] as training phase followed by new quantization architecture based testing phase to perform compression. The proposed technique is helpful in client server environment whereby the volume of the data sent has to be lessening to minimize the time taken for transmission. Here, FastICA algorithm is used in calculating estimated matrix W and separated matrix X. Figure 8 outlines the block diagram of proposed EICA image compression.

Image enhancement is the popular and the most widely known technique of image processing. Many images like medical images, satellite images, aerial images and even real life photography suffer from noise and poor contrast. Image enhancement algorithms offer a wide variety of approaches for modifying images to achieve visually acceptable images. The choice of such techniques is a function of the specific task, image content, observer characteristics, and viewing conditions. The point processing methods are most primitive, yet essential image processing operations and are used primarily for contrast enhancement [15]. Enhancement techniques improve the quality of the image view, blurring, noise and increasing contrast and improve the borders and sharpness of the image.

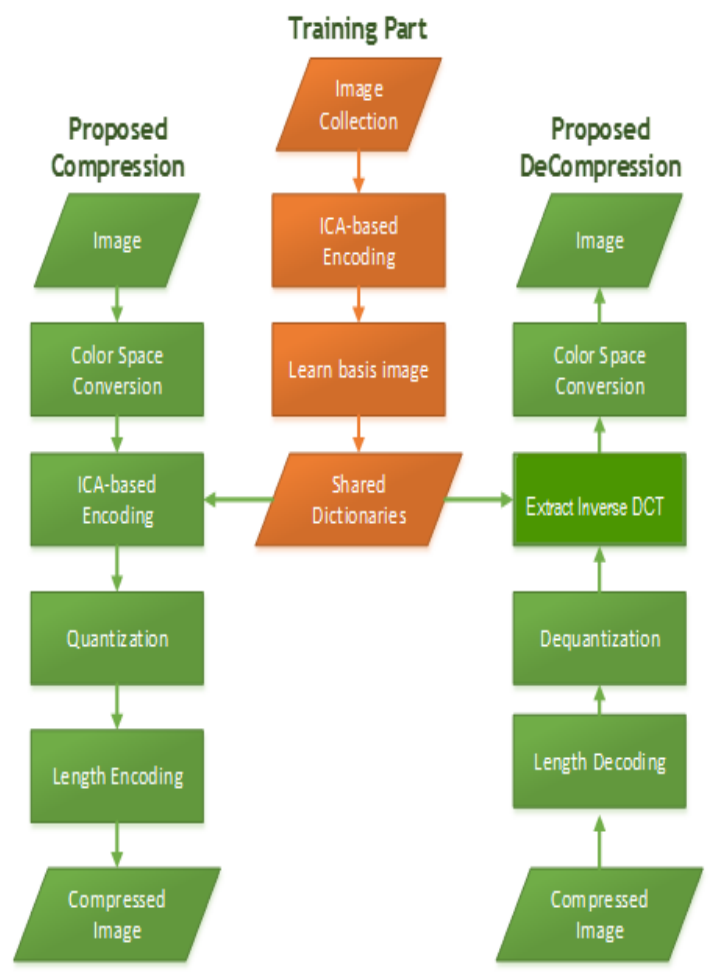

Figure 8: Block Diagram of Proposed Image Compression

The first step in the EICA process is breaking an image into its color components which are R, G and B. After that, each color component is divided into $8 \times 8$ image sub-blocks. Each sub-block is a training set, after mean removal and sphering or whitening by the principal component analysis (PCA). FastICA is used in the calculation of the estimated matrix wi and separating matrix xi for every sub-block image.

After $\mathrm{W}$ and $\mathrm{X}$ are calculated, the arithmetic coding changes the data into binary format and send $\mathrm{W}$ and $\mathrm{X}$ matrix information to the receiver and again reconstructing $\mathrm{W}$ and $\mathrm{X}$ utilizing all sub-block information and multiplying the $\mathrm{W}$ and $\mathrm{X}$ to reconstruct the image. In this proposed method, the EICA based new developed quantization architecture as zero coefficient percentage setting obtained by one of the methods as follows:

1- Mode 1: Quantization architecture via zero quantized coefficients percentage setting.

2- Mode 2: Quantization architecture via adaptive zero quantized coefficients percentage.

The functional scheme of the suggested EICA based on new quantization architecture using training and testing platforms are illustrated in Figure 3.9. 


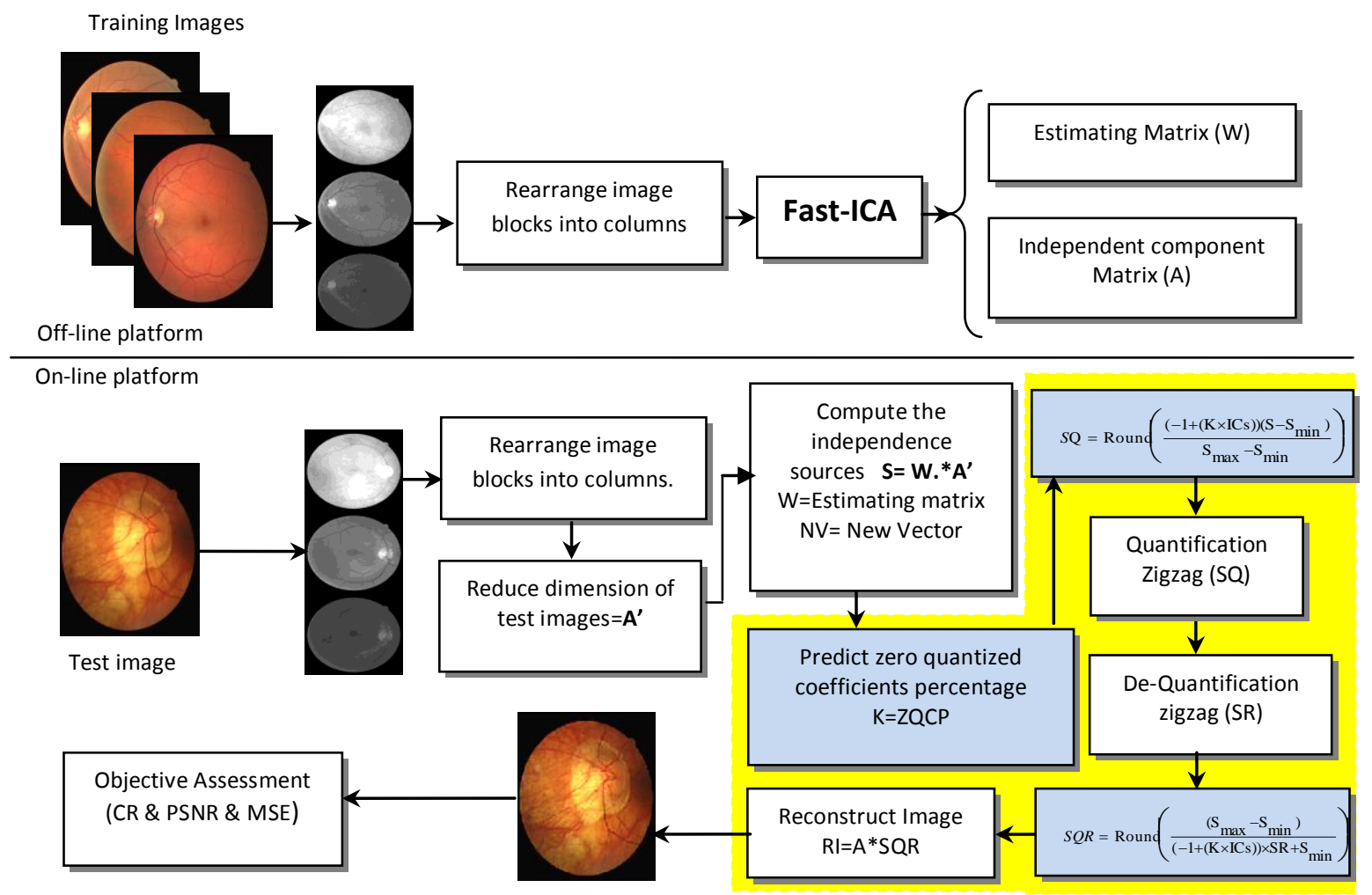

Figure 9: Details explanation of the EICA using training and testing platforms

- Quantization architecture via adaptive zero quantized coefficients percentage

In this proposed quantization architecture, a collection of medical data is handled off-line to generate archived data which is saved in a relational database. A fastICA is used to train images and estimate matrix (W) and independent component matrix (A) in the database is determined. Only the estimated matrix and the separating matrix of the trained images are returned to the user. The following are the steps for studying the basis images which are performed once: -

1) Off-line platform: In the off-line platform, the outline of the medical image compression technique presented includes studying ICA bases from the training images by utilizing FastICA as follows:

1.1 Obtain the intensity matrix for the entire training medical images.

1.2 Separate the RGB components of training medical images.

\begin{tabular}{|l|l|}
\hline 2.1 & Select test images and apply the steps from (1.1) until (1.4) as in the off-line platform \\
\hline 2.2 & $\begin{array}{l}\text { Reduce dimension of resulting single matrix of the test image by remove the mean from vectors using } \\
\text { PCA. }\end{array}$ \\
\hline 2.3 & $\begin{array}{l}\text { Compute the independence source (S) by multiplying the resulting new vector (A') from step } 2.2 \text { and the } \\
\text { separating matrix (W) from training stage: } \mathrm{S}=\mathrm{W}^{*} \mathrm{~A}^{\prime}\end{array}$ \\
\hline 2.4 & $\begin{array}{l}\text { Then, perform zero quantized coefficients percentage (ZQCP) prediction model, only features that have } \\
\text { significant values returned and others are set to zero }\end{array}$ \\
$\qquad \begin{array}{l}\text { New ICA matrix (SQ) achieving quantification stage as follows: } \\
\text { SQ }\end{array}$ \\
Where, $\mathrm{K}$ is the adaptive resulting value form the ZQCP prediction model, ICs is the optimized
\end{tabular}

1.3 Dividing every RGB component into 8x 8 subblocks whereby every block is a column of matrix $\mathrm{X}_{0}$

1.4 Rearrange image blocks into columns with the selected number of Independent Components and convert the resulting cell array into a single matrix

1.5 Obtain and save the estimated separating matrix (W) and the corresponding mixing matrix (A) by applying FastICA.

Next, the On-line platform then performed compression to the test image via quantization architecture via zero quantized coefficients percentage (ZQCP) prediction model as indicated in Figure 9 (highlighted yellow color box).

2) On-line platform: In the online-platform, the following steps are examined for the new quantization architecture proposed for EICA compression 


\begin{tabular}{|l|l|}
\hline & independent component \\
\hline 2.6 & Data Encoding achieved by the zigzag sequence of block size 8 by 8. \\
\hline$S Q R=\operatorname{Round}\left(\frac{\left(\mathrm{S}_{\text {max }}-\mathrm{S}_{\text {min }}\right)}{(-1+(\mathrm{K} \times \mathrm{ICs})) \times \mathrm{SR}+\mathrm{S}_{\text {min }}}\right)$ \\
\hline 2.8 & $\begin{array}{l}\text { Achieving de-quantification stage, a new ICA matrix }(\mathrm{SQR}) \text { as follows: } \\
\text { from the off-line platform. }\end{array}$ \\
\hline
\end{tabular}

\section{- ZQCP Prediction Model}

In this stage, the main concept behind the EICA based on new strategies for data compression by enhancing ICA with a new quantization architecture based zero quantized coefficient percentage (ZQCP) prediction model. In order to accomplish this objective:-

- First, the usage of mode 1 as zero coefficient percentage setting with variable threshold factor values has to be investigated and a conclusion of its effect on the compression and quality of the medical data will be drawn

- Second, a new quantization architecture for ZQCP is designed using statistical pixel-level (SPL) elements extraction and artificial neural networks (ANN's) model as a classifier to predict ZQCP value.

- Third, compute the new ICA matrix (SQ) based the predicted ZQCP value and succeeding quantification stage.

- $\quad$ Fourth, compute the new ICA matrix (SQ) based the predicted ZQCP value and succeeding quantification and de-quantification stages.

- Fifth, compute the new matrix (SQR) based the predicted $\mathrm{ZQCP}$ value and reconstruct image.

- Sixth, results of the experiment are obtained and examined to access the performance of the model performance. This assessment is carried out through visual interpretation and quantitative calculation based on the difference in the measurement of PSNR and MSE between the input and the images obtained from modes 1 and 2 .

The primary steps used in the proposed algorithm with ZQCP prediction in the medical data are: feature extraction, target generation, ZQCP model construction and assessment. Figure 10 illustrates the functional flow of the proposed zero quantized coefficients percentage (ZQCP) prediction model.

ZQCP prediction: Training stage ZQCP prediction: Testing stage

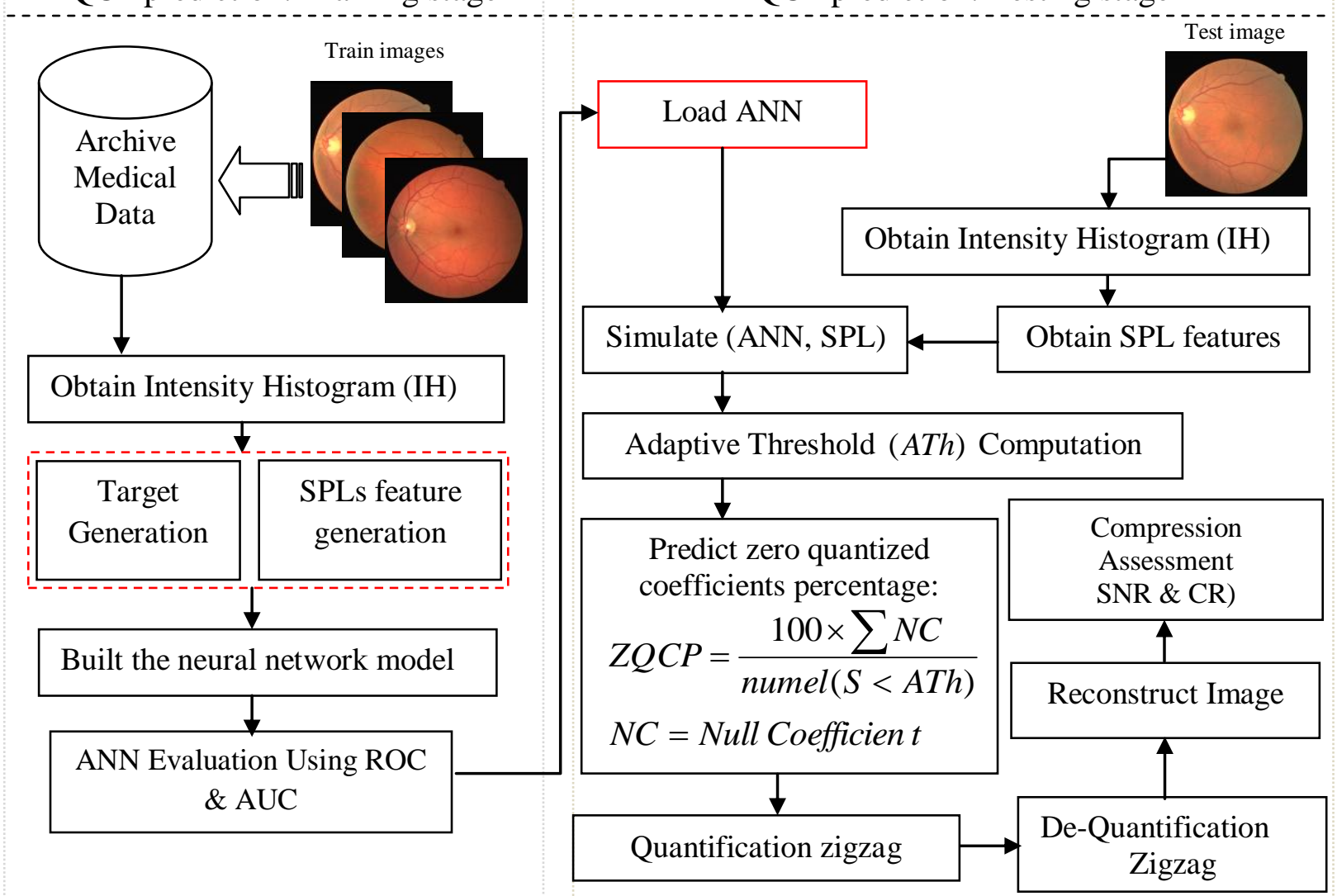

Figure 10 Functional flow of the proposed zero quantized coefficients percentage (ZQCP) prediction model 


\section{- Statistical pixel-level (SPL)}

Features which are noted by [16], are obtained from a particular rectangular area within the RG images. Next, the SPL features are computed using intensity histograms of their coresponding extracted area. Intensity histogram denotes a graphic illustration of the number of pixels in an image at different levels of intensity in an area. When the image intensity histogram is obtained from the cropped area, information of the grey-level pixels is utilised to compute the SPL features such as energy, mean, variance, kurtosis, skewness and entropy.

1) Equation 1 is used to calculate the mean of the gray values of all pixels in the area where

$$
\begin{aligned}
& L \in[0, L-1] \\
& M=\frac{1}{n} \sum_{i=0}^{L-1}\left(r_{i} p\left(r_{i}\right)\right)
\end{aligned}
$$

Whereby $p\left(r_{i}\right)$ denotes the probability and $n\left(r_{i}\right)$ denotes quantity of occurrences of the gray value $\left(r_{i}\right)$ in the area $n$ denote the total number of pixels in the area.

2) The variance for gray value pixel is:

$$
V=\sum_{i=0}^{L-1}\left(p\left(r_{i}\right)\left(r_{i}-m\right)\right)^{n}
$$

Whereby $n=2, n=3$ and $n=4$ are the variance at the third and the fourth instants of the area.

3) Energy of the gray value pixels is obtained from:

$$
E=\sum_{i=0}^{L-1}\left(p\left(r_{i}\right)\right)^{2}
$$

4) Entropy measures data representation through the distribution of gray values and is obtained from:

$$
E n=\sum_{i=0}^{L-1} p\left(r_{i}\right) \log _{2}\left(r_{i}\right)
$$

5) Skewness (f5) measures symmetry whereby the distribution of a set of data $y_{1}, y_{2}, \ldots y_{n}$ is symmetrical if the left area of the central point is identical to the right area

$$
S k=\sum_{i=1}^{n} \frac{\left(y_{i}-\bar{y}\right)^{3}}{(n-1) S^{3}}
$$

Whereby $\bar{y}$ represents mean $S$ represents standard deviation and $n$ represents the number of data points

6) Kurtosis measures whether the distribution of a set of data $y_{1} y_{2}, \ldots y_{n}$ has a peak or are relatively distributed compared to a normal distribution and is calculated as:

$$
K u=\sum_{i=1}^{n} \frac{\left(y_{i}-\bar{y}\right)^{4}}{(n-1) S^{4}}
$$

The SPL element vector is obtained from the image intensity histogram, which represents the medial data. Let denote the SPL vector as $f=[M, V, E, E n, S k, K u]$. The normalized vector $f_{\text {nor }}$ for each image in the data set is contempleted to create the featured vector database as follows.

$$
f_{\text {nor }}=\frac{f}{\max (f)}
$$

\section{- Target generation}

To create the ANN-MLP training phase and SPLs model elements, the targets that serve as the class label need to be computed using histogram technique. This technique divides the intensity histogram into ten bins (intervals) which are equally spaced. Each bin will contain the number of score occurrences in a set of data. The following steps are utilised to create the ZQCP value using binning histogram technique.

1. Obtain the intensity histogram of medical data images.

2. Determine the value for every histogram bin, get the histogram account and bin location.

3. Divide the resulting image histogram into ten equally spaced interval bins. The first histogram bin value is 0 to $1 /(n=10)$ and the subsequent histograms have positive increase of step $1 / n$ till 1 .

4. Determine the area of every bin using its histogram account and location.

5. Sort the measured areas according to descending order together with their corresponding indexes.

6. Then, the maximum intensity area will be reflected as the target for the processes image.

\section{TRANSMISSION SIMULATION}

Image compression algorithm is internationally recognized as a vital tool for decreasing communication bandwidth and saving transmitting power. Image compression algorithms should reproduce good quality image after compression at low bit rates. Therefore, previously discussed compression algorithms were investigated for transmission simulation.

\subsection{Simulation Schema}

Figure 13 shows simulation schema details of medical data transmission through Wi-Fi protocol. The first step is data conversion, which converts medical data (e.g., images and video frames) to bits in binary-arranged stream form. DBPSK modulation was performed on data transmitted through Wi-Fi protocol. Chip factor up-sampling and filtering were applied to modulation data stream before transmitting data. During data transmission, the channel consists of multipath fading with additive white Gaussian noise (AWGN). AWGN was employed to represent actual conditions, in which noise occurs in Wi-Fi transmission because of EMI with other apparatus or electric systems. The inverse operation was employed at the receiver. 


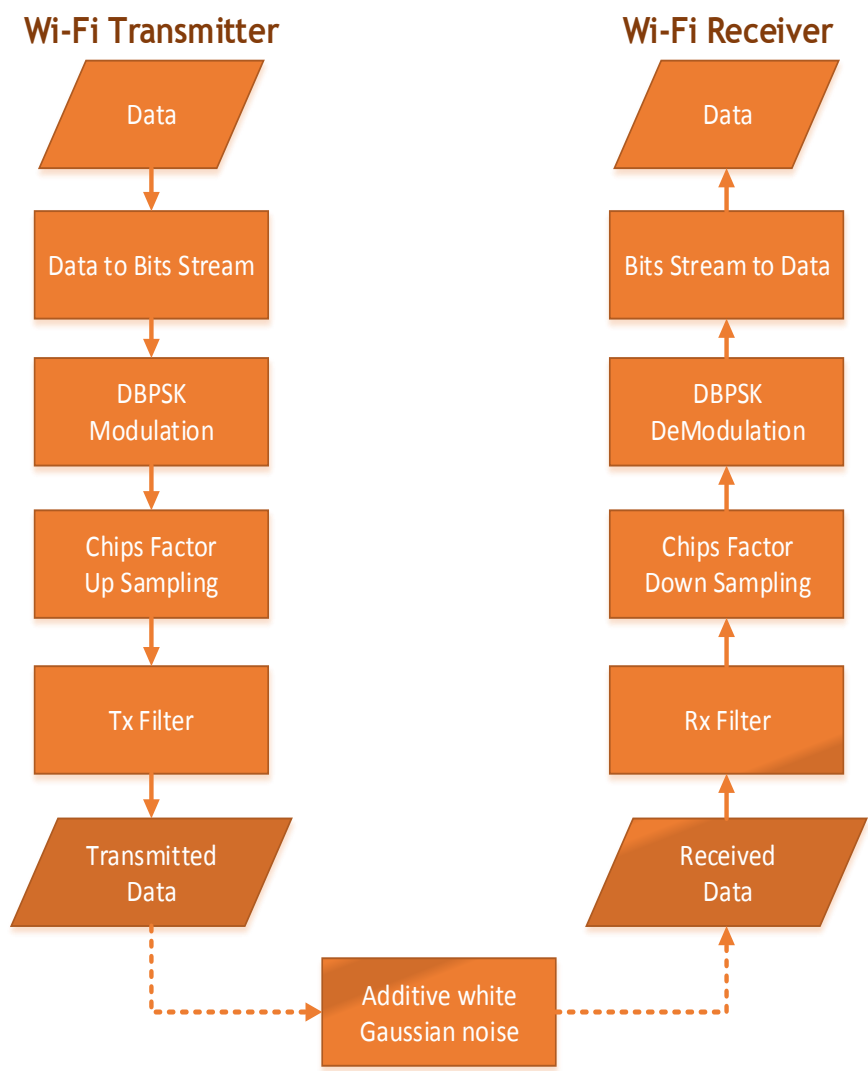

Figure 13: Simulation schema of medical data transmission over $\mathrm{Wi}-\mathrm{Fi}$ protocol

\subsection{Data Bits Stream Converter}

Medical data transmitted in bit format are converted into bit stream before entering Wi-Fi simulation stage. In this research, medical images were used as one sample of the data type. Bit streams of medical images were extracted from pixel intensity values containing R, G, and B channels. Data were arranged as R, G, and B pixels. The 8-bit pixel intensity is represented by 1-byte data that consist of 8-bit binary data. In the case of 16-bit pixel intensity, data bits are represented by 2-byte data or 16-bit binary data. Figure 14 shows an example of bit stream conversion on the ultrasound image, in which each pixel is represented by three layers of the 8-bit pixel value [17].

In case of 8 bits, each pixel was initially converted to 8-bit binary data and then arranged as a series of data. Length of data series depends on dimensions of medical data. In this research, the header of image file was excluded from simulation stage because the focus was to determine effects of image compression on Wi-Fi transmission protocol.

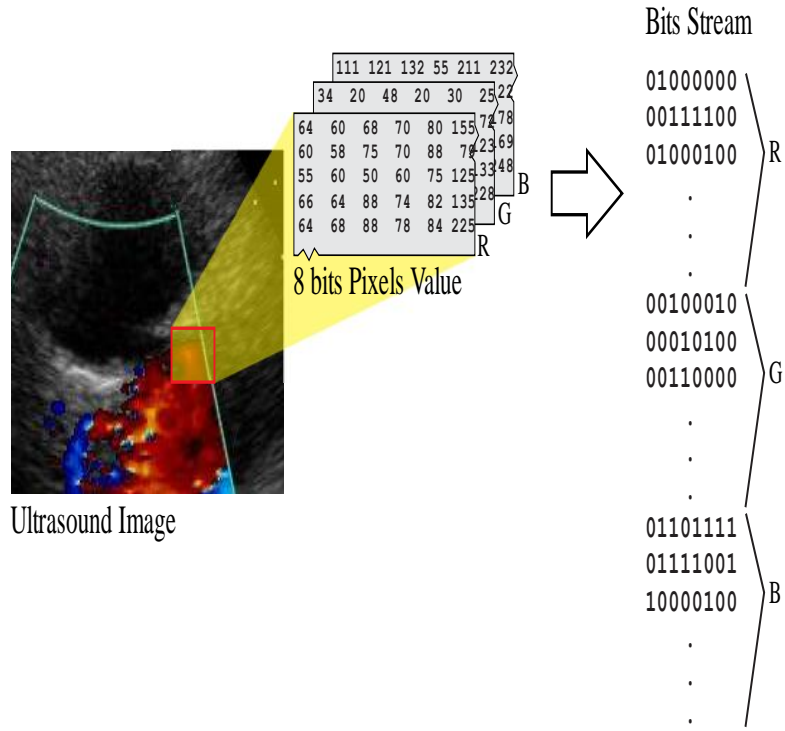

Figure 14: Example of bits stream conversion on medical image with RGB channel

\subsection{DBPSK Modulation}

WLAN standard IEEE 802.11b utilizes diverse PSKs, which is dependent on required data rate. This standard utilizes DBPSK at the basic rate of $1 \mathrm{Mbit} / \mathrm{s}$. DQPSK was utilized to provide an extended rate of $2 \mathrm{Mbit} / \mathrm{s}$. QPSK was employed but was combined with the corresponding code keying to reach $5.5 \mathrm{Mbit} / \mathrm{s}$ and full rate of $11 \mathrm{Mbit} / \mathrm{s}$. IEEE $802.11 \mathrm{~g}$ features eight data rates with high-speed WLAN standard, that is, $6,9,12,18,24,36,48$, and $54 \mathrm{Mbit} / \mathrm{s}$. The 6 and $9 \mathrm{Mbit} / \mathrm{s}$ modes utilized OFDM modulation, where respective subcarriers were BPSK-moderated. OFDM with QPSK was utilized with 12 and $18 \mathrm{Mbit} / \mathrm{s}$ modes. The four fastest modes utilized OFDM with quadrature amplitude modulation forms [18].

Amplified signals in transmit $(\mathrm{Tx})$ and receive $(\mathrm{Rx})$ directions eliminated effects of lossless information between Wi-Fi transmitter and $\mathrm{Wi}-\mathrm{Fi}$ receiver, allow mounting, and benefitted data transmitted and received over WLANs.

- In Tx direction, the signal was amplified to ensure that transmitted power was maximized, was not lost in the Wi-Fi band, and remained unaffected by AWGN.

- In Rx direction, the signal was amplified to ensure that received data featured the best quality. However, strength and clarity were reduced.

\subsection{Gaussian Noise}

WGN was added to the transmitted signal, assuming $0 \mathrm{~dB}$ of input power. Noise affected transmitted data, which is in binary format. Values of some transmitted data changed from 1 to 0 or from 0 to 1 because of WGN. In this simulation, medical data stream was divided into packet bit sizes[19]. Therefore, noise affected each packet data transmitted through Wi-Fi. Figure 15 shows an example of WGN effect on bitstream using various levels of noise. WGN changed values of bits from 0 to 1 or vice versa [20] and affected medical data by changing some information in images or signals transferred through the Wi-Fi connection. 
1110000001101111010000000000000000000000

(a)

1111000000111111011001001010010100110000

(b)

1110001001111111110000000011101100000110

(c)

Figure 15: Example of Gaussian noise: (a)

original bit stream, (b) bit stream with $5 \mathrm{~dB}$

Gaussian noise, and (c) bit stream with $10 \mathrm{~dB}$ Gaussian noise

\subsection{Performance Metrics}

Assessment of performance comprised two parts. First, performance results connected by similar data were transferred and acknowledged, representing an error bit. Second, performance results were considered the similarity between the original image before and after transmission calculated utilizing PSNR[21]. The goal of image quality measure was to provide quantitative scores in capturing similarity (or contrariwise, the error) between the original (reference image) and distorted versions [22].

\section{i. $\quad$ Error Bits}

Error bits between transmitted and received data were used to evaluate the proposed scheme, which sends compression medical data through Wi-Fi [23]. In this research, errors were simulated by WGN, which was added during data transmission. Error bits were computed as follows:

$$
\text { error }=\frac{\sum_{i=0}^{N} b_{i} \otimes b_{i}{ }^{\prime}}{N}
$$

Where; $b_{i}$ and $b_{i}{ }^{\prime}$ refer to bit streams before and after transmission, respectively.

\subsubsection{MSE and PSNR}

MSE is an engineering term for the ratio between the maximum possible power of a signal and corrupting noise power affecting representation fidelity. Given that numerous signals feature extensive dynamic ranges, expression of MSE

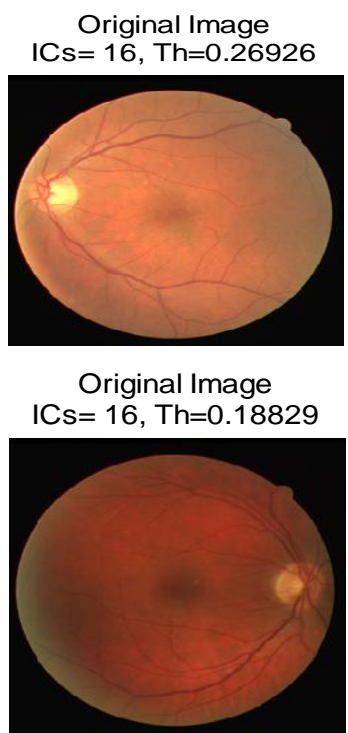

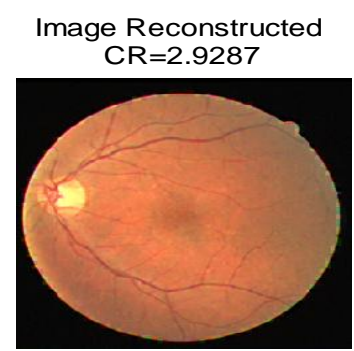

Image Reconstructed $\mathrm{CR}=2.8534$

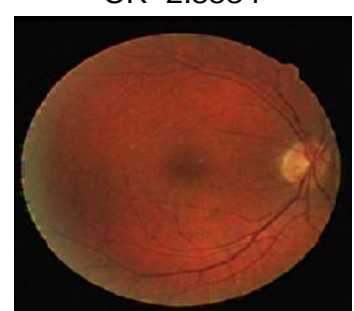

is frequently used in terms of logarithmic decibel scale. PSNR and MSE are most frequently utilized as lossy compression codecs to measure reconstruction quality (for example, to compress image). In this case, signals represented the new data, and the noise was an error introduced by compression.

$$
\operatorname{MSE}(a, b)=\frac{1}{N} \sum_{i=1}^{N}\left(a_{i}-b_{i}\right)^{2}
$$

High PSNR and low MSE usually indicate high-quality reconstruction[24]. Mathematical definition of MSE between two images (a) and (b) is expressed as follows:

Where; $N$ corresponds to the number of pixels in images, and $a_{i}$ and $b_{i}$ indicate pixel values in $i^{\text {th }}$ position of sample vectors.

PSNR was used to evaluate image quality by comparing received and original images. PSNR is computed as follows:

$$
P S N R=10 \log \frac{\text { peakval }^{2}}{M S E}
$$

Where; peakval is obtained from the range of image data types using $n$ bits of image.

\section{CONCLOSION}

This paper presents the proposed methodology and research framework, including the compression module for data compression and decompression using four different algorithms, that is, DCT, SPIHT, JPEG200, and the proposed EICA compression algorithm. Simulation transceiver module was also presented for simulation of transmitted and received data over WLAN IEEE 802.11b. Initial performance results are regarded as the similarity between the original image and resulting image before and after compression with the four investigated algorithms by computing the PSNR, CR and MSE. The second performance results related to transmitted and received data similarity are examined using bit error rate (BER) and PSNR in the simulation TRS module 


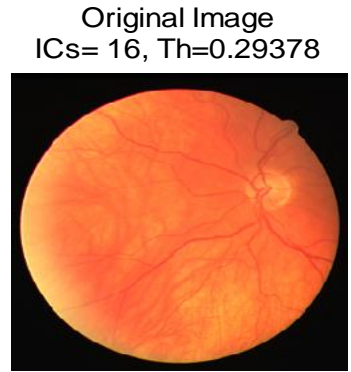

Original Image

$I C s=16, T h=0.30926$

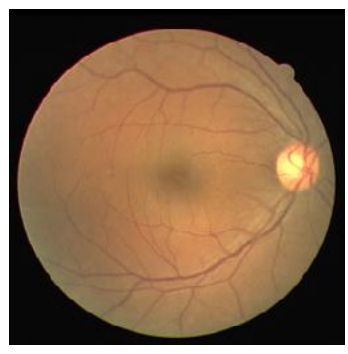

Original Image $\mathrm{ICs}=16, \mathrm{Th}=0.50046$

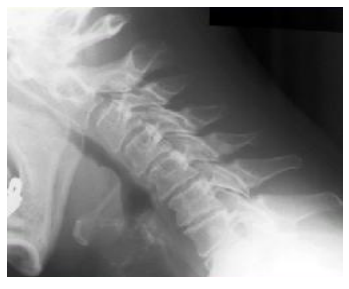

Original Image $\mathrm{ICs}=16, \mathrm{Th}=0.35481$

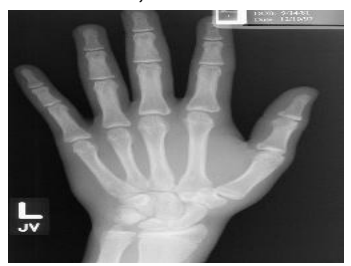

Original Image ICs=16, Th=0.48556

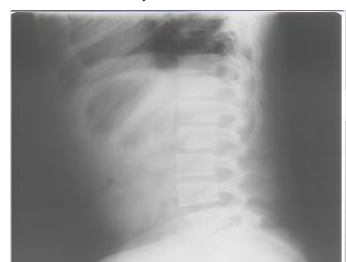
ICs $=16, \mathrm{Th}=0.037908$

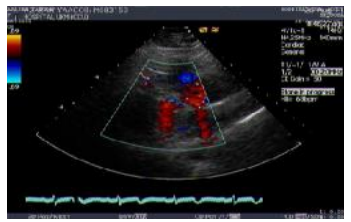

Image Reconstructed $\mathrm{CR}=2.7925$

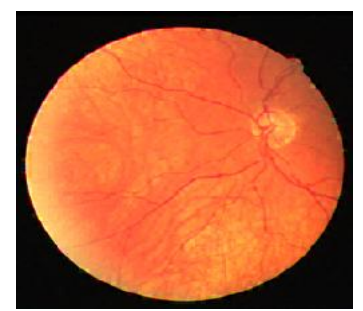

Image Reconstructed $\mathrm{CR}=2.8678$

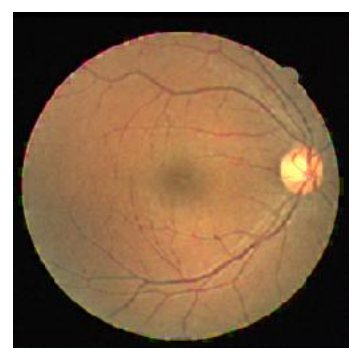

Image Reconstructed $\mathrm{CR}=3.1566$

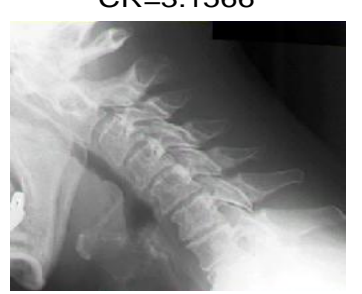

Image Reconstructed $\mathrm{CR}=3.192$

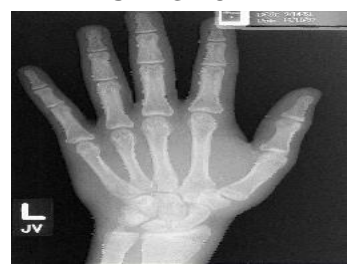

Image Reconstructed $\mathrm{CR}=2.9135$

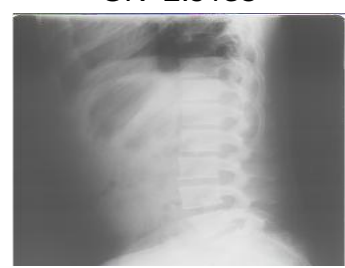

Image Reconstructed

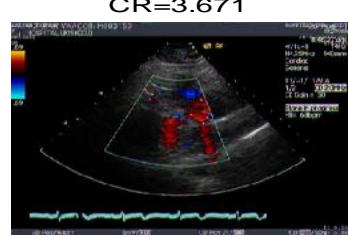

Error Image (PSNR, MSE) (34.0853, 0.00039036)

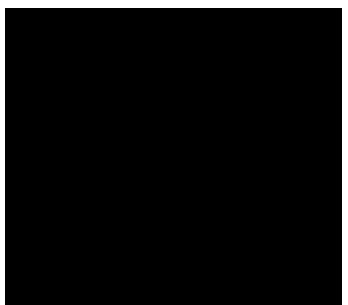

Error Image (PSNR, MSE) (33.3632, 0.00046097)

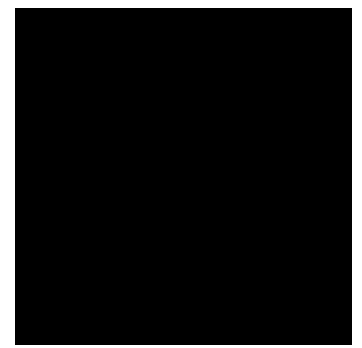

Error Image (PSNR, MSE) (38.8041, 0.0001317)

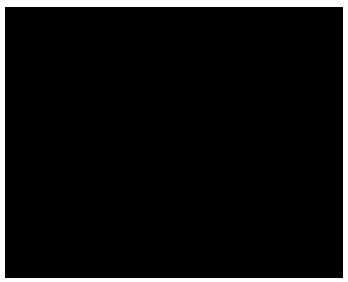

Error Image (PSNR, MSE) (32.8862, 0.0005145$)$

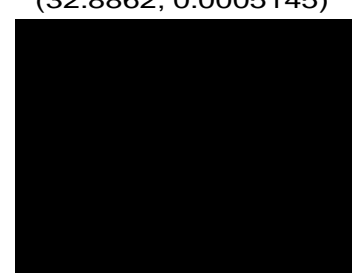

Error Image (PSNR, MSE) (39.3727, 0.00011554)

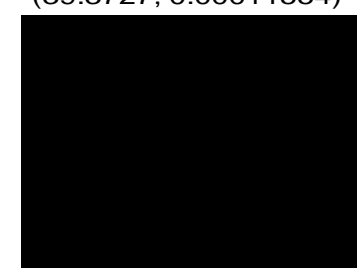

Error Image (PSNR, MSE) (33.4443, 0.00045245)

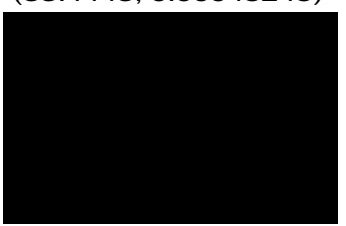



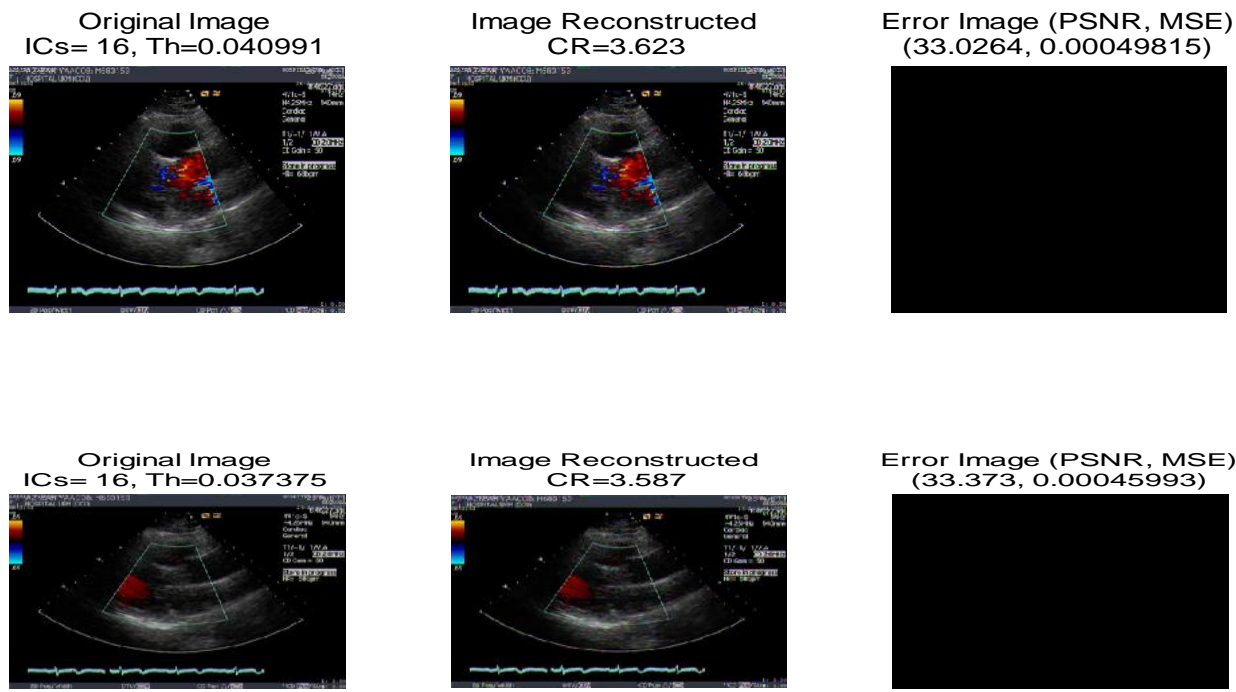

\section{ACKNOWLEDGMENTS}

This research was funded by the Ministry of Higher Education Libya and Elmergib University

\section{REFERENCES}

[1] S. T. Wong and D. A. Tjandra, "A digital library for biomedical imaging on the Internet," IEEE Communications Magazine, vol. 37, pp. 84-91, 1999.

[2] M. A. Algaet, Z. A. B. M. Noh, A. S. B. HASAN BASARI, A. S. Shibghatullah, A. A. Milad, and A. Mustapha, "SIMULATION OF MEDICAL DATA COMPRESSION AND TRANSMISSION THROUGH WLANS," Journal of Theoretical \& Applied Information Technology, vol. 82, 2015.

[3] W. D. Bidgood Jr, S. C. Horii, F. W. Prior, and D. E. Van Syckle, "Understanding and using DICOM, the data interchange standard for biomedical imaging," Journal of the American Medical Informatics Association, vol. 4, pp. 199-212, 1997.

[4] T. Kalinski, R. Zwönitzer, M. Roßner, H. Hofmann, A. Roessner, and T. Guenther, "Digital Imaging and Communications in Medicine (DICOM) as standard in digital pathology," Histopathology, vol. 61, pp. 132134,2012

[5] L. Zhang, A. Afanasyev, J. Burke, V. Jacobson, P. Crowley, C. Papadopoulos, et al., "Named data networking," ACM SIGCOMM Computer Communication Review, vol. 44, pp. 66-73, 2014.

[6] J. Jones, A. Sargsyan, Y. Barr, S. Melton, D. Hamilton, S. Dulchavsky, et al., "Diagnostic ultrasound at MACH 20: retroperitoneal and pelvic imaging in space," Ultrasound in medicine \& biology, vol. 35, pp. 10591067, 2009.

[7] P. R. Oliveira, H. Morimitsu, and E. F. Tuesta, "Using visual metrics to selecting ICA basis for image compression: a comparative study," in Ibero-American Conference on Artificial Intelligence, 2010, pp. 80-89.

[8] M. A. Algaet, Z. A. B. Muhamad Noh, A. S. Shibghatullah, A. A. Milad, and A. Mustapha, "Telemedicine and its application in healthcare management: Telemedicine management," 2014.

[9] A. Hyvärinen, J. Karhunen, and E. Oja, Independent component analysis vol. 46: John Wiley \& Sons, 2004.

[10] S. Masood, M. Sharif, M. Yasmin, M. Raza, and S.
Mohsin, "Brain image Compression: A brief survey," Research Journal of Applied Sciences, Engineering and Technology, vol. 5, pp. 49-59, 2013.

[11] F. Huang, J. Huang, and Y. Q. Shi, "Detecting double JPEG compression with the same quantization matrix," IEEE Transactions on Information Forensics and Security, vol. 5, pp. 848-856, 2010.

[12] M. A. Algaet, Z. A. M. Noh, A. S. H. Basari, A. S. Shibghatullah, A. A. Milad, and A. Mustapha, "A review of service quality in integrated networking system at the hospital scenarios," Journal of Telecommunication, Electronic and Computer Engineering (JTEC), vol. 7, pp. 61-69, 2015.

[13] M. N. Islam, "System and method for a wireless medical communication system," ed: Google Patents, 2007.

[14] A. Hyvarinen, "Fast and robust fixed-point algorithms for independent component analysis," IEEE transactions on Neural Networks, vol. 10, pp. 626-634, 1999.

[15] R. Maini and H. Aggarwal, "A comprehensive review of image enhancement techniques," arXiv preprint arXiv:1003.4053, 2010.

[16] R. C. Gonzalez and R. E. Woods, "Digital image processing prentice hall," Upper Saddle River, NJ, 2002.

[17] A. Mustapha, A. Oulefki, M. Bengherabi, E. Boutellaa, and M. A. Algaet, "Towards nonuniform illumination face enhancement via adaptive contrast stretching," Multimedia Tools and Applications, vol. 76, pp. 2196121999, 2017.

[18] A. Doufexi, S. Armour, M. Butler, A. Nix, D. Bull, J. McGeehan, et al., "A comparison of the HIPERLAN/2 and IEEE 802.11 a wireless LAN standards," IEEE Communications magazine, vol. 40, pp. 172-180, 2002.

[19] M. A. Algaet, Z. A. B. M. Noh, A. S. Shibghatullah, A. A. Milad, and A. Mustapha, "Provisioning quality of service of wireless telemedicine for e-health services: A review," Wireless Personal Communications, vol. 78, pp. 375-406, 2014.

[20] T. Richardson and R. Urbanke, "The renaissance of Gallager's low-density parity-check codes," IEEE Communications Magazine, vol. 41, pp. 126-131, 2003.

[21] M. A. Algaet, Z. A. B. M. Noh, A. S. Shibghatullah, and A. A. Milad, "Provisioning quality of service of wireless telemedicine for e-health services," in 2013 IEEE Conference on Information \& Communication 
Technologies, 2013, pp. 199-202.

[22] J. Galbally and S. Marcel, "Face anti-spoofing based on general image quality assessment," in 2014 22nd International Conference on Pattern Recognition, 2014, pp. 1173-1178.

[23] M. A. Algaet, Z. A. B. M. Noh, A. S. B. H. Basari, A. S. Shibghatullah, A. A. Milad, A. B. Abugharsa, et al., "Development of Robust Medical Image Transmission via Wi-Fi IEEE 802.11 b in the Hospital Area," Wireless Personal Communications, vol. 95, pp. 1617-1634, 2017.

[24] M. Gupta and A. K. Garg, "Analysis of image compression algorithm using DCT," International Journal of Engineering Research and Applications (IJERA), vol. 2, pp. 515-521, 2012. 